\title{
Efecto del número de goteros y la frecuencia de riego sobre la producción, calidad del fruto y productividad del agua en una plantación de alta densidad de manzanos
}

\author{
J.R. Gispert ${ }^{1}$, F. Ramírez de Cartagena ${ }^{2,{ }^{*}}$, J.M. Villar ${ }^{3}$, J. Rufat ${ }^{4}$ y I. Batlle ${ }^{1}$ \\ 1 Institut de Recerca i Tecnologia Agroalimentàries (IRTA), Mas de Bover, Ctra Reus-El Morell, Km 3.8, \\ 43120 Constantí (Tarragona), España \\ 2 Universitat de Girona, Departament d'Enginyeria Química, Agrària i Tecnologia Agroalimentària, \\ Campus Montilivi, 17071 Gerona, España \\ 3 Universitat de Lleida, Departament de Medi Ambient i Ciències del Sòl. Av. Alcalde Rovira Roure, \\ 191, 25198 Lérida, España \\ 4 Institut de Recerca i Tecnologia Agroalimentàries (IRTA), Programa Ús Eficient de I Aigua, Alcalde \\ Rovira Roure, 191, 25198 Lérida, España
}

\begin{abstract}
Resumen
Se realizó durante tres años consecutivos un ensayo para determinar el efecto del número de emisores por planta y el intervalo de riego sobre los parámetros de producción, calidad y productividad del agua en manzanos de diez años, cv 'Golden Delicious' sobre portainjerto EM9, cultivados en sistema intensivo en un suelo aluvial del noreste de España. El experimento consistió en aplicar la misma dosis de agua, determinada de acuerdo con las necesidades de riego, a través de tres diseños agronómicos distintos (1, 2 y 3 goteros autocompensantes de $8 \mathrm{Lh}^{-1}$ por árbol, separados $1,0,8$ y 0,4 m, respectivamente), manteniendo el tiempo de riego constante y variando la frecuencia de riego en cada diseño $(3,6$ y 9 días de media, respectivamente). No se observó ninguna diferencia entre los tres diseños para ninguna de las variables analizadas $(P<0,05)$, excepto el índice de almidón. La falta de diferencias en los parámetros de producción entre tratamientos puede atribuirse en parte a la semejanza del patrón hídrico que finalmente se desarrolló en los distintos diseños, como consecuencia del idéntico volumen de agua aplicado y de la franja de humedad continua que se formó en la hilera del cultivo, debido a la proximidad entre goteros. En las condiciones del ensayo, el diseño agronómico con 1 emisor por árbol y frecuencia de riego 3 días permitió alcanzar producciones similares a las obtenidas con 2 o 3 emisores y frecuencias de riego menores, con ahorro en el coste económico de la instalación.
\end{abstract}

Palabras clave: Producción intensiva de manzano, riego por goteo, diseño agronómico, uso del agua de riego, volumen de suelo húmedo, coste instalación goteo.

\begin{abstract}
Effect of the number of drippers and irrigation frequency on production, fruit quality and water productivity in a high-density apple orchard

Field experiments were conducted during three consecutive years to determine the effect of the number of drippers per plant and irrigation frequency on the production, quality and water productivity parameters in ten year old apple trees, cv 'Golden Delicious' on rootstock EM9, grown in an intensive system
\end{abstract}

\footnotetext{
* Autor para correspondencia: francisco.ramirez@udg.edu http://doi.org/10.12706/itea.2017.002
} 
with high-density planting in an alluvial soil of north-eastern Spain. The experiment consisted in applying the same dose of water, determined according the irrigation water needs, through three different agronomic designs (1, 2 and 38 Lh-1 pressure-compensating drippers per tree), maintaining a constant irrigation time and varying the irrigation frequency ( 3,6 and 9 days on average). No differences were observed among the three treatments for any of the variables analyzed $(P<0.05)$, except for starch index. The lack of differences in production parameters between treatments, can probably be attributed in part to the standardization of the hydric pattern eventually developed in the three designs, due to the same water amount of water applied and also because the formation of a strip of continuous moisture along the crop row caused by the proximity between drippers. In the test conditions, the agronomic design with 1 dripper per tree and 3 days frequency achieved similar yields to those obtained with 2 or 3 drippers and lower irrigation frequencies, thus reducing installation costs.

Key words: Intensive apple production, drip irrigation, agronomical design, irrigation water use, wet soil volume, drip installation cost.

\section{Introducción}

En la mayoría de países productores de manzana, la producción se ha incrementado a causa del establecimiento de modelos intensivos más que al aumento de la superficie de cultivo. Lograr aumentos en el rendimiento a través del incremento de la densidad de plantación provoca grandes cambios en el diseño y manejo de las plantaciones frutales (Raffo e Iglesias, 2004). Los sistemas con alta densidad de plantación permiten acelerar la entrada en producción, reducen la altura y volumen de las plantas facilitando las labores de cosecha y poda, permiten disminuir la cantidad de agroquímicos aplicada, aumentan la homogeneidad en las plantas y posibilitan la reducción de costes. Oster y Wichelns (2003) apuntaron que los modelos tecnológicos futuros deberían considerar un riguroso equilibrio entre producción agrícola, sustentabilidad de los recursos naturales agua y suelo, y el uso energético. En efecto, actualmente la intensificación del cultivo del manzano está creando la necesidad de desarrollar y aplicar técnicas que sean más efectivas para obtener la mayor productividad por unidad de superficie, con un mejor uso de los recursos, especialmente agua y energía tanto desde un punto de vista de interés medioambiental como económico.
Las necesidades de agua, la frecuencia y el tiempo de riego son importantes criterios de diseño a considerar en la elección entre métodos de riego en manzano (Kadayifçi et al., 2010). Neilsen et al. (1995) indicaron que la alta frecuencia de riego mejoraba el crecimiento vegetativo, pero el efecto sobre las concentraciones de nutrientes foliares o los intercambios químicos del suelo era menor que al aplicar una frecuencia más baja. Earl y Jury (1977), analizando el movimiento del agua en un suelo cultivado regado con un emisor aislado, observaron que el riego semanal en relación al riego diario mojaba más superficie de suelo (horizontal y vertical) y por tanto de volumen radicular, por lo que las raíces en el caso semanal estaban más extendidas y en el caso diario ocupaban un volumen más reducido, resultando producciones más altas en el riego semanal. En manzanos, Levin et al. (1979) también encontraron que el riego diario con emisores de $4 \mathrm{Lh}^{-1}$ en un suelo basáltico de $60 \mathrm{~cm}$ de profundidad generó una distribución de la humedad del suelo más reducida que cuando se regó semanalmente, siguiendo la distribución de raíces un patrón paralelo a la distribución de humedad, con aumento de la densidad radicular en la zona mojada.

El volumen de suelo mojado es la variable más importante tanto en el diseño como en el 
posterior manejo del riego localizado, puesto que es el principal factor que determina la distribución de raíces. Levin et al. (1973) indican que la relación entre el contenido de agua del suelo y la aireación fue determinante en el desarrollo radicular de manzanos sometidos a diferentes regímenes de riego. La distribución del agua en el suelo y la forma y tamaño del volumen de suelo húmedo (VSH) en riego localizado varía notablemente dependiendo del tipo de suelo, del caudal del emisor y del tiempo de riego, así como del número de emisores por árbol. La determinación en campo del frente de humedad y sus dimensiones características como profundidad o radio mojado es compleja y supone una inversión importante en tiempo y coste económico, de manera que la estimación de estas variables a partir de ecuaciones empíricas obtenidas a través de modelizaciones de procesos físicos (Simunek et al., 2006; Arbat et al., 2008, 2013) constituye una alternativa interesante. Rumayor y Bravo (1991) estudiaron el efecto sobre el vigor, la producción y los parámetros de calidad del fruto en manzano 'Golden Delicious' y 'Top Red Delicious' de 3 niveles de VSH (inundación, goteo y microaspersión), corregidos cada uno de ellos mediante 3 coeficientes $(0,2,0,3$ y 0,5$)$ respecto a los valores de lectura de un tanque evaporimétrico clase $\mathrm{A}$. La producción por árbol fue significativamente más elevada usando microaspersores, pero los parámetros de calidad no mostraron ninguna diferencia significativa entre los diferentes sistemas de riego o porcentajes de VSH. Rufat (2003) comparó diferentes estrategias de riego sobre las variedades de manzano 'Top Red','Early Red One' y 'Smoothe', así como dos sistemas de riego (goteo y microaspersión) con similar suministro de agua (570 $\mathrm{mm}$ ) y diferente porcentaje de VSH. El experimento mostró una mayor producción total acumulada de manzana con el sistema de goteo, aunque sin diferencia significativa respecto al sistema con microaspersión y los va- lores de producción estuvieron más relacionados con el número de frutos por árbol que con el peso individual del fruto. La firmeza del fruto fue más baja en árboles regados usando riego por goteo que con microaspersión. Sin embargo, este comportamiento estuvo inversamente relacionado con el índice de almidón en fruto, cuyos mayores valores se obtuvieron con goteo.

Zur (1996), para fines de diseño de riego localizado, indica la necesidad de establecer un valor mínimo del volumen de suelo a humedecer. Keller y Bliesner (1990) proponen como un objetivo razonable en el diseño para cultivos plantados a un marco amplio, como árboles, humedecer entre un tercio y dos tercios de la sección horizontal del sistema radicular, es decir, conseguir un porcentaje de suelo mojado entre el $33 \%$ y $66 \%$. Zhao et al. (2012) utilizan el porcentaje de suelo húmedo en superficie propuesto por Amin y Ekhmaj (2006) para recomendar, en condiciones de clima extremadamente árido, el porcentaje de superficie húmeda del $32 \%$ como el óptimo durante los primeros años en las transformaciones de riego por superfície a goteo en cultivo de peral. Gispert et al. (2012) introducen el concepto de volumen potencial de exploración radicular (VPER) como valor de referencia de los volúmenes de suelo húmedo en riego localizado, con la finalidad de conseguir una mejor aproximación a la realidad de la plantación. Para ello ensayaron 2 estrategias de riego (total y deficitario controlado) y 5 porcentajes de volumen de suelo húmedo dentro de cada estrategia en olivo cv. 'Arbequina', observando que el porcentaje del $59 \%$ en riego deficitario controlado fue el que generó una mayor producción de aceite, sin embargo en riego total no se encontraron diferencias significativas entre los distintos porcentajes de volumen de suelo mojado. Bajo el mismo concepto de volumen potencial de exploración radicular Gispert et al., (2015) recomendaron el 33\% de VSH para el avellano, cv. 'Negret'. 
En plantaciones intensivas, con marcos de plantación que suponen separaciones entre plantas alrededor de $1 \mathrm{~m}$ o incluso menores, suelen producirse solapamientos entre los bulbos de humedad formados por los goteros, que acaban definiendo una franja contínua a lo largo de la hilera de plantas, lo que debe tenerse en consideración en la determinación del VSH.

López et al. (1992) consideran que es importante determinar el número de emisores por planta tomando como base el área humedecida, la seguridad de riego y el costo. Girona et al. (2008) observan una reducción drástica de la evaporación del agua del suelo al disminuir la superficie mojada como consecuencia de pasar de 2 a 1 gotero por planta en riego deficitario controlado de manzanos cv 'Golden Smoothe', recomendando la eliminación de un gotero por planta como mejor opción para reducir las pérdidas por evaporación frente a la disminución de la frecuencia de riego.

El área y el volumen de suelo húmedo final alcanzado están en relación con el número de goteros instalados y éste con el coste de la instalación, puesto que un porcentaje importante de la inversión inicial corresponde a las tuberías laterales y emisores, que constituyen un costo fijo. Por consiguiente, la determinación del número de goteros por planta y su ubicación es una de las decisiones más importantes en el diseño del riego por goteo ya que condiciona la frecuencia de riego y el porcentaje de suelo mojado, variables determinantes en el establecimiento de las condiciones de humedad y oxígeno adecuadas en la zona radicular. Por otra parte la frecuencia de riego practicada, que es un coste variable vinculado con la operación del sistema, también está relacionada con el número de emisores y tendrá consecuencias sobre el volumen de suelo húmedo definido. Consecuentemente, la configuración de la instalación en relación al número de goteros y la frecuencia de riego practicada en el manejo de la plantación, son dos de las variables más significativas asociadas a la producción del cultivo y los costes derivados.

El objetivo de este trabajo es analizar y evaluar como afecta el número de goteros por planta y la frecuencia de riego aplicada al comportamiento productivo, vegetativo y de calidad del fruto en manzano, con la finalidad de establecer una recomendación basada en criterios de rendimiento del cultivo y de ahorro económico en la instalación de riego por el número de goteros utilizado.

\section{Material y métodos}

\section{Parcela experimental}

El experimento tuvo una duración de 3 años consecutivos (1998-2000) y se realizó en una parcela de $650 \mathrm{~m}^{2}$ situada en la Fundación Mas Badia (IRTA) en 'La Tallada d'Empordà' (Gerona, noreste de España), plantada de manzano (Malus domestica Borkh), cultivar 'Golden Delicious' de 10 años de edad sobre portainjerto EM9 con una densidad de 2.857 árboles ha-1 $(3,5 \mathrm{~m} \times 1 \mathrm{~m})$. Los árboles, formados en eje central, fueron podados de la misma forma y tenían un volumen medio de copa de $8,5 \mathrm{~m}^{3}$. Las copas de los árboles contiguos se solapaban, siendo el radio sombreado por árbol de 1,26 $\mathrm{m}$ y el porcentaje de área sombreada del $72 \%$.

La parcela estaba localizada en un suelo aluvial clasificado como Typic Xerorthents (Soil Survey Staff, 2010), con características muy similares en todo el perfil respecto a $\mathrm{pH}(7,6-$ $7,7), \mathrm{CE}_{1: 5}(0,52-0,57 \mathrm{dS} / \mathrm{m})$, materia orgánica $(1,31-0,75 \%)$, textura (franco-arenosa de 0 a $30 \mathrm{~cm}$ y arenosa-franca de 30 a $60 \mathrm{~cm}$ ), carbonato cálcico equivalente $(1,52-3,43 \%)$, caliza activa (imperceptible) y contenido volumétrico medio de agua $(17,28 \%$ a $-20 \mathrm{kPa}$; $4,75 \%$ a $-1.500 \mathrm{kPa}$ ). 
La información climática necesaria para el desarrollo del ensayo, precipitación y evapotraspiración, se obtuvo de la Estación Meteorológica ubicada en la Fundación Mas Badía, situada a $50 \mathrm{~m}$ de la parcela experimental y perteneciente a la XEMA (Red de Estaciones Meteorológicas Automáticas), gestionada por el Servicio Meteorológico de Cataluña. Durante el periodo experimental (1998-2000) la temperatura media anual fue de $15,5^{\circ} \mathrm{C}$.

Necesidades de riego

Les necesidades de agua de riego fueron calculadas semanalmente mediante la ecuación:

$$
N t=\frac{E T C-P e}{E f}
$$

siendo $\mathrm{N}_{\mathrm{t}}$, las necesidades totales de riego $(\mathrm{mm}) ; \mathrm{ET}_{\mathrm{C}^{\prime}}$ la evapotranspiración del cultivo $(\mathrm{mm}) ; \mathrm{P}_{\mathrm{e}^{\prime}}$ la lluvia efectiva $(\mathrm{mm})$ y $\mathrm{E}_{\mathrm{f}}$ la eficiencia de riego.

La evapotranspiración del cultivo (ETC) (Allen et al., 1998) se determinó a partir de la evapotranspiración de referencia $\left(\mathrm{ET}_{0}\right)$, calculada con el método de Penman-Monteith, corregido por el coeficiente de cultivo $\left(K_{C}\right)$ y el coeficiente de reducción $\left(\mathrm{K}_{\mathrm{e}}\right)$. Los coeficientes de cultivo $\left(\mathrm{K}_{\mathrm{C}}\right)$ utilizados fueron los del manzano, de acuerdo a Doorenbos y Pruitt (1988), [Abril $(0,45)$, Mayo $(0,55)$, Junio $(0,80)$, Julio $(0,90)$, Agosto $(0,9)$, Septiembre $(0,85)$ y Octubre $(0,65)]$. El K $\mathrm{K}_{\mathrm{e}}$ fue considerado igual a 1 ya que el cultivo cubría una superficie mayor del $70 \%$ del marco de plantación (Fereres et al., 1981). La precipitación efectiva se estimó en el $70 \%$ de la precipitación total y no se consideraron las precipitaciones inferiores a $10 \mathrm{~mm}$ (Smith, 1988). Finalmente, las necesidades totales de riego se determinaron considerando una eficiencia de riego del $90 \%$, de acuerdo con la prueba de uniformidad realizada en la misma parcela (coeficiente de uniformidad 95\%) y la eficiencia de percolación estimada a partir de
Keller y Bliesner (1990). Los ensayos de uniformidad se realizaron al inicio de cada campaña de riego mostrando un valor alto y muy parecido entre años debido al buen mantenimiento de la instalación, la calidad del agua utilizada, la topografia llana y la utilización de goteros autocompensantes.

La temporada de riego comprendió los meses de abril hasta septiembre, ambos incluidos.

\section{Tratamientos de riego}

Se establecieron tres tratamientos de riego, correspondientes a la instalación de 1, 2 y 3 emisores por árbol. En cada fila de árboles se instaló 1 ramal de riego a 0,5 m del tronco, con emisores autocompensantes de $8 \mathrm{Lh}^{-1}$ insertados. Los emisores se colocaron linealmente, de manera que para el caso de 1 emisor, éste se situó a $40 \mathrm{~cm}$ del tronco, con una separación entre emisores de $1 \mathrm{~m}$; en el caso de 2 emisores por planta, se situaron uno a cada lado del tronco y separados 80 $\mathrm{cm}$; y en el diseño con 3 emisores, uno se colocó próximo al tronco y los dos restantes a $40 \mathrm{~cm}$ del tronco cada uno de ellos, siendo 40 $\mathrm{cm}$ la separación entre emisores.

El tiempo de riego se estableció en 3 horas, para todos los tratamientos y durante todo el periodo experimental, con una intención doble. Por una parte, para cada gotero individual, este planteamiento contribuyó a la definición de volúmenes de suelo húmedos similares en los distintos tratamientos puesto que en todos ellos el caudal del gotero fue el mismo. Por otra parte, puesto que la programación del riego se efectuó de acuerdo a los requerimientos hídricos de la semana anterior, la pauta para satisfacer las necesidades de agua de riego obligaba a variar la frecuencia de riego, variable objetivo del estudio.

De esta forma la frecuencia de riego varió de acuerdo con el número de emisores instalados y la demanda evapotranspirativa sema- 
nal, correspondiendo la frecuencia más elevada al tratamiento con menor número de goteros y la frecuencia menor al mayor número de goteros.

El número de riegos semanales se determinó como el cociente entre las necesidades de agua de riego de la semana anterior y el volumen de agua aportada durante el tiempo de riego, es decir 24,48 y $72 \mathrm{~L}$ para los tratamientos con 1, 2 y 3 goteros, respectivamente. En el caso en que el número de riegos fuera un número decimal, se redondeaba al número entero más próximo. Cada tratamiento de riego tenía instalado en cabecera un contador volumétrico (Arad Multijet 1", Israel; unidad legible más pequeña 0,05 L; exactitud $\pm 2 \%$ a $\pm 5 \%$ según caudal circulante) que permitía conocer los pequeños déficits o excesos de agua que podían producirse debido al redondeo de la frecuencia de riego $u$ otras causas y que se corregían al final de la semana uniformizando los distintos tratamientos.

\section{Diseño experimental}

El diseño experimental fue en bloques al azar y 3 repeticiones para cada tratamiento $(1,2$ y 3 emisores). Cada una de las 9 parcelas elementales constó de 5 árboles control y un árbol guarda entre parcelas. El ensayo involucró un total de 60 árboles, 45 de los cuales controlados y 15 considerados árboles guarda.

\section{Determinación y formación del VSH en las pruebas previas de campo con emisores aislados}

Para determinar la forma y tamaño del VSH generado por cada emisor aislado se empleó el equipo medidor del volumen de suelo húmedo (EMVSH) (Gispert et al., 2012, 2015). Las lecturas de resistividad eléctrica se consideraron para una temperatura del suelo alrededor de los $22^{\circ} \mathrm{C}$. La prueba de campo para determinar la forma media del VSH se realizó en la propia parcela con un emisor autocompensante de $8 \mathrm{Lh}^{-1}$ y tres repeticiones. El contorno del frente de humedad se definió por los puntos donde la diferencia de resistividad eléctrica fue de $3.000 \Omega \mathrm{m}$ o el $3 \%$ de diferencia de humedad. Utilizando este método se definió el radio de la superficie húmeda a $30 \mathrm{~cm}$ de profundidad y el volumen de suelo húmedo $\left(\mathrm{m}^{3}\right)$. Se efectuaron mediciones del contenido de agua del suelo a 1, 2 y 3 horas del inicio del riego. A las 3 horas del riego, valor correspondiente al tiempo de riego fijado en los ensayos, la forma del VSH en las tres repeticiones fue muy similar con un radio húmedo de $62 \mathrm{~cm}$ a $30 \mathrm{~cm}$ de profundidad y una profundidad mojada de 110 cm (Figura 1). Por otra parte se efectuó otra medida del contenido de agua en el suelo en los bulbos de humedad del ensayo a las $24 \mathrm{~h}$ después de finalizar el riego de $3 \mathrm{~h}$ de duración. Los resultados mostraron que la redistribución fue poco acusada, puesto que la profundidad mojada aumentó aproximadamente un $15 \%$ y el radio mojado alrededor del $10 \%$, comportamiento esperable en una textura marcadamente arenosa como la del ensayo.

El cilindro de suelo situado bajo la copa del árbol, que tiene por base la propia proyección de la copa y por altura la profundidad estimada de las raíces, se usó para calcular el VPER. Un total de 10 mediciones aleatorias permitieron determinar el radio medio de la proyección de la copa (1,26 m). La profundidad radicular considerada fue de $1 \mathrm{~m}$, que se evaluó mediante tres muestreos aleatorios bajo la copa de tres manzanos, realizando sendas calicatas de $1,5 \mathrm{~m}$ de profundidad y 1 $\mathrm{m}$ de ancho. Si bien la mayor densidad de raíces se encontró entre 50 y $60 \mathrm{~cm}$ de profundidad, la observación de raíces importantes hasta $1 \mathrm{~m}$ aconsejó adoptar este valor en los cálculos del VPER. El área sombreada media de los manzanos $\left(4,98 \mathrm{~m}^{2}\right)$ se obtuvo asumiendo un volumen esférico de la copa y considerando el radio de proyección de la 


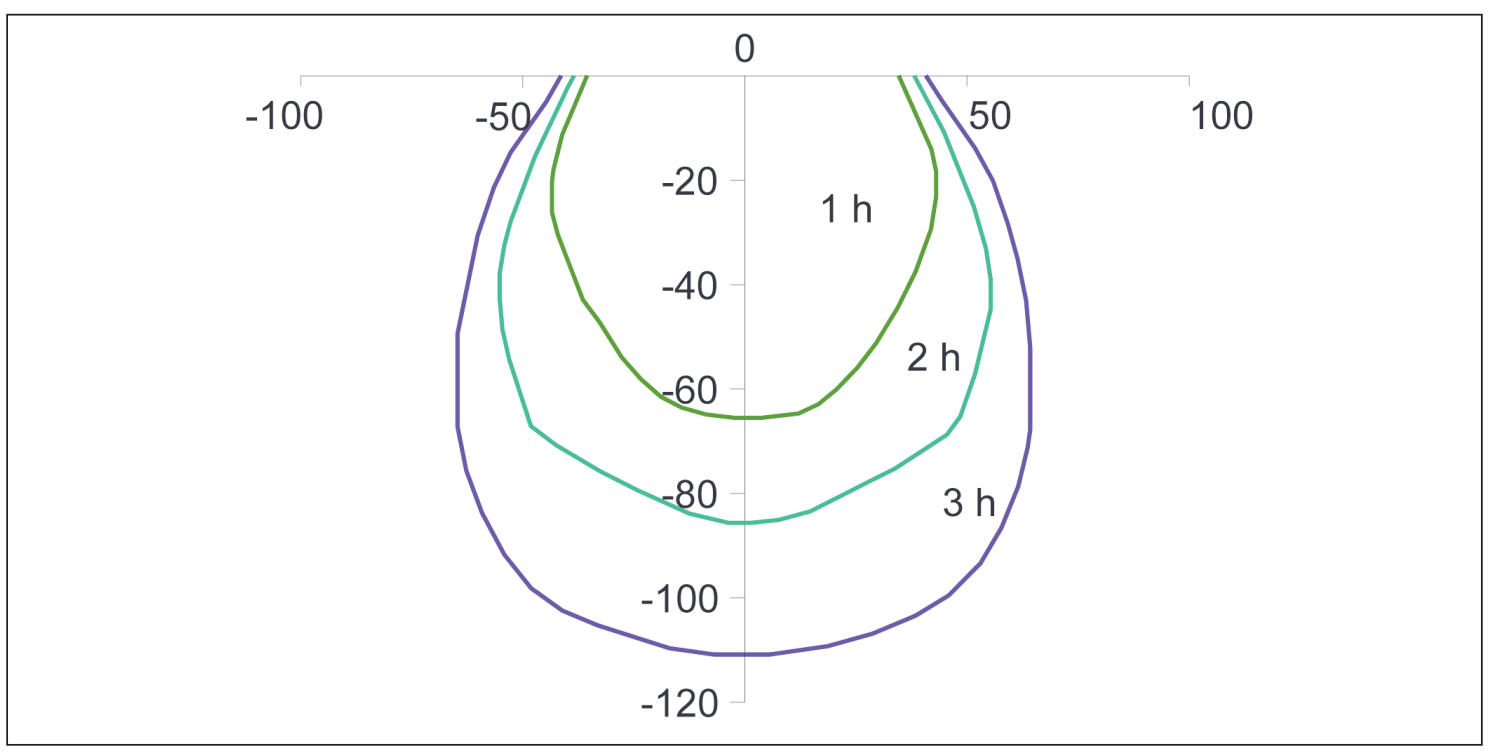

Figura 1. Forma y dimensiones $(\mathrm{cm})$ de la sección correspondiente al volumen de suelo húmedo generado por un emisor de caudal $8 \mathrm{Lh}^{-1}$ para los tiempos de riego de

1,2 y 3 horas en la parcela experimental.

Figure 1. Shape and dimensions $(\mathrm{cm})$ of the section corresponding to wet soil volume generated with a dripper of $8 \mathrm{Lh}^{-1}$ with run times of 1, 2 and 3 hours in the experimental plot

copa. El VPER $\left(4,98 \mathrm{~m}^{3}\right)$ se obtuvo a partir del producto del área sombreada por la profundidad radicular $(1 \mathrm{~m})$. La realización previa de las pruebas de campo con emisores aislados, sin solape entre los bulbos de humedad desarrollados, permitió conocer la correspondencia entre el número de emisores utilizados y los porcentajes de VSH conseguidos, que fueron de 22, 44 y $66 \%$ VPER, respectivamente para 1, 2 y 3 emisores. En estas condiciones de trabajo la profundidad de raíces fue un $10 \%$ inferior a la profundidad de suelo mojado, estando por tanto dentro del rango admitido en el diseño agronómico del riego localizado (López et al., 1992).

\section{Parámetros productivos evaluados}

En la época de recolección y durante los años 1998, 1999 y 2000, se evaluó la producción total y comercial (calibre $>70 \mathrm{~mm}$ ) contro- lando la carga individual de cada uno de los 5 árboles de cada parcela elemental. Para el control de calidad, se utilizó una máquina industrial para clasificar el fruto recolectado en los diferentes árboles de cada parcela elemental. Asimismo, el peso medio del fruto (g) se calculó al dividir la producción total obtenida en cada parcela elemental respecto al número de frutos. El número medio de frutos por árbol (N) se obtuvo del conteo realizado en cada árbol y parcela elemental.

\section{Parámetros vegetativos evaluados}

Para asegurar que el tamaño inicial de los árboles era similar en todos los tratamientos, se calculó el volumen de copa al inicio del experimento. Nueve árboles fueron evaluados, uno de cada parcela elemental. La forma de la copa se consideró similar a un elipsoide con diferentes radios (altura vertical de la copa, dis- 
tancia horizontal en la dirección de la fila de los árboles, distancia horizontal perpendicular a la fila). Las mediciones fueron realizadas durante tres años consecutivos usando una cinta plegable de $5 \mathrm{~m}$ (Lufkin Ultralok) y para las mediciones verticales se utilizó una mira telescópica de $8 \mathrm{~m}$ (Telefix). Para calcular el crecimiento anual, se seleccionaron y marcaron dos brotes en la dirección Norte-Sur, sobre cada uno de los 5 árboles correspondientes a cada parcela elemental.

Parámetros de calidad evaluados

Los parámetros de calidad se evaluaron a partir de muestras de fruto recogidas en septiembre y durante los años 1999 y 2000. La firmeza del fruto se evaluó con un penetrómetro (Fruit Pressure Tester, FT 327), eligiendo 10 frutos de cada parcela elemental. Se determinó el índice de almidón mediante colorimetría por tinción de yodo (prueba de Lugol) sobre 10 manzanas partidas y recolectadas en cada parcela elemental.

\section{Productividad del agua de riego}

La productividad del agua $\left(\mathrm{P}_{\mathrm{a}} \mathrm{kg} \mathrm{m}^{-3}\right)$ se definió por la relación entre la producción total de manzana comercializable $\left(P, \mathrm{~kg} \mathrm{ha}^{-1}\right)$ respecto al volumen de agua de riego aplicado $\left(\mathrm{U}, \mathrm{m}^{3} \mathrm{ha}^{-1}\right)$ :

$$
P a=\frac{P}{U}
$$

\section{Análisis estadístico}

Los resultados fueron estadísticamente analizados utilizando el SAS System Program (SAS, User's Guide 1996). El modelo planteado permitió analizar el efecto de cada tratamiento $(1,2,3$ emisores) sobre cada uno de los parámetros considerados (producción, crecimiento vegetativo, calidad del fruto, productividad del agua), así como comparar los distintos tratamientos para cada parámetro.
El modelo estadístico usado fue:

$$
Y_{i j k}=\mu+A_{j}+E N_{k}+e_{i j k}
$$

$\mathrm{Y}_{\mathrm{ij}}$ observación del $\mathrm{i}^{\text {th }}$ árbol del año $\mathrm{j}^{\text {th }}$ sometido al número de goteros $\mathrm{k}^{\text {th }}\left(\mathrm{EN}_{\mathrm{j}}\right) ; \mu$, valor medio de la variable observada; $A_{j}$, efecto año (1998, 1999, 2000); $E_{k^{\prime}}$ efecto del número de goteros por planta $(1,2$ y 3$)$; i, 1, 2, 3 y e, error.

Se realizó un análisis de la varianza y un test de separación de medias (test de Duncan), para un nivel de significación $\alpha=0,05$.

\section{Resultados y discusión}

Evapotranspiración, pluviometría y volumen de agua de riego aplicado

La evapotranspiración media anual del período experimental fue muy similar para todos los años con un valor medio de $869,7 \mathrm{~mm}$, mientras que la lluvia efectiva fue de 491,2 mm, siendo el primer año el más seco con aproximadamente el $18 \%$ menos de lluvia que el año medio (Tabla 1). Durante el periodo de riego hubo poca variabilidad entre los diferentes años para los valores de evapotranspiración de referencia y de precipitación efectiva, que además en este periodo supusieron aproximadamente el $70 \%$ y el $34 \%$ de los valores anuales, respectivamente. Para cada año del ensayo la dosis de riego aplicada en cada tratamiento fue muy parecida, presentando coeficientes de variación de 1,3\%,2,1\% y $1,3 \%$ para los años 1998, 1999 y 2000 respectivamente, que garantizan la homogeneidad en el volumen de agua aportado en cada uno de los tratamientos. Para la temporada de riegos, la dosis de riego aplicada más la lluvia efectiva estimada fue de $584,9 \mathrm{~mm}$ como valor medio del conjunto de años del ensayo, cantidad muy similar a la indicada por Drake y Evans, (1997) y Rufat (2003) con un valor medio de $570 \mathrm{~mm}$ para 'Golden Smoothe' en riego localizado en Lérida (España). 
Tabla 1. Evapotranspiración de referencia $\left(E T_{0}\right)$ y lluvia efectiva $\left(P_{e}\right)$ para cada año en el periodo experimental y en el periodo de riego (abril a septiembre) y dosis de riego anual para cada año y tratamiento en el periodo de riego (1998-2000)

Table 1. Reference evapotranspiration $\left(E T_{0}\right)$ and effective rainfall $\left(P_{\mathrm{e}}\right)$ for each year during the experimental period and during the irrigation period (April to September) and irrigation dose for each year and treatment during the irrigation period (1998-2000)

\begin{tabular}{|c|c|c|c|c|}
\hline & 1998 & 1999 & 2000 & Media \\
\hline \multicolumn{5}{|c|}{ Periodo experimental } \\
\hline $\mathrm{ET}_{0}(\mathrm{~mm})$ & 845,9 & 884,8 & 878,4 & $869,7(20,8)$ \\
\hline$P_{t}(m m)$ & 405,1 & 551,9 & 516,6 & $491,2(76,6)$ \\
\hline \multicolumn{5}{|c|}{ Periodo de riego } \\
\hline $\mathrm{ET}_{0}(\mathrm{~mm})$ & 627,2 & 609,2 & 604,1 & $613,7(12,5)$ \\
\hline$P_{e}(m m)$ & 156,0 & 184,0 & 166,7 & $168,9(14,1)$ \\
\hline \multicolumn{5}{|c|}{ Dosis de riego } \\
\hline 1 gotero & 452,6 & 399,4 & 414,3 & $422,1(27,4)$ \\
\hline 2 goteros & 440,6 & 397,7 & 406,3 & $414,9(22,6)$ \\
\hline 3 goteros & 445,7 & 384,0 & 403,4 & $411,0(31,5)$ \\
\hline Media & $446,3(6,0)$ & $393,7(8,4)$ & $408,0(5,6)$ & $416,0(24,3)$ \\
\hline
\end{tabular}

Desviaciones típicas se indican entre paréntesis.

\section{Frecuencia de riego}

El valor medio del número de riegos por temporada fue de 61, 30 y 20 para el diseño con 1, 2 y 3 goteros respectivamente.

En la Tabla 2 se muestra la frecuencia de riego para los distintos tratamientos y cada uno de los años y meses del periodo de riego. Los valores medios y desviaciones típicas de la frecuencia de riego durante el periodo experimental fueron de $2,8(2,0), 5,7(4,2)$ y 8,6 $(6,6)$ días para los tratamientos con 1,2 y 3 goteros respectivamente.

\section{Producción de manzana}

Durante el período experimental, la producción media total y comercial no fue significativamente diferente entre los tratamientos comparados. El riego con 2 goteros por plan- ta mostró valores más altos en cada año del ensayo, aunque sin diferencias significativas respecto a los restantes tratamientos (Tabla 3). Los resultados de producción total y comercial fueron muy similares a los obtenidos por Rufat (2003) que tampoco mostraron diferencias significativas al aumentar el porcentaje de volumen de suelo mojado.

El peso medio del fruto y el número de frutos por árbol no mostraron diferencias estadísticas significativas entre los diversos tratamientos (Tabla 4), aunque también los valores más altos se alcanzaron para el riego con 2 goteros por planta. La distribución de la calidad del fruto no está influida únicamente por el volumen de agua aplicada, sino por otros factores como: carga de frutos por árbol, vigor del portainjerto, nivel nutricional y factores ambientales (Thalheimer et al., 2000). 
Tabla 2. Frecuencia de riego media (días) para cada mes y año del periodo experimental

Table 2. Average irrigation frequency (days) for each month and year of the experimental period

\begin{tabular}{lcrrrrrr}
\hline Año & Abril & Mayo & Junio & Julio & Agosto & Septiembre & Media \\
\hline 1998 & & & & & & & \\
Tratamiento 1 & - & 3,1 & 1,9 & 1,6 & 2,1 & 6,0 & $2,3(1,2)$ \\
Tratamiento 2 & - & 6,2 & 3,8 & 3,1 & 3,9 & 15,0 & $4,6(2,8)$ \\
Tratamiento 3 & - & 10,3 & 6,0 & 4,4 & 6,2 & 15,0 & $7,0(3,4)$ \\
1999 & & & & & & & \\
Tratamiento 1 & 7,5 & 7,8 & 2,3 & 1,8 & 1,9 & 7,5 & $3,1(2,3)$ \\
Tratamiento 2 & 15,0 & 15,5 & 5,0 & 3,9 & 3,9 & 15,0 & $6,5(4,6)$ \\
Tratamiento 3 & 30,0 & 31,0 & 7,5 & 5,2 & 6,2 & 30,0 & $10,2(9,3)$ \\
2000 & & & & & & & \\
Tratamiento 1 & 15,0 & 3,9 & 2,7 & 2,2 & 1,6 & 5,0 & $3,0(1,1)$ \\
Tratamiento 2 & 30,0 & 7,8 & 5,0 & 4,4 & 3,4 & 10,0 & $6,1(2,1)$ \\
Tratamiento 3 & 30,0 & 15,5 & 7,5 & 6,2 & 5,2 & 15,0 & $9,2(3,9)$ \\
\hline
\end{tabular}

Desviaciones típicas se indican entre paréntesis.

Tabla 3. Producción total y comercial de cosecha de manzana y producción total acumulada en los diferentes tratamientos (número de goteros) durante el período experimental

Table 3. Total and commercial production of apple yield and total accumulated production in the different treatments (number of drippers) during the experimental period

\begin{tabular}{|c|c|c|c|c|c|c|c|c|}
\hline \multirow[b]{2}{*}{$\mathrm{N}^{\circ}$ goteros } & \multicolumn{4}{|c|}{ Total (kg ha-1) } & \multicolumn{4}{|c|}{ Comercial $^{1}(\%)$} \\
\hline & 1998 & 1999 & 2000 & Media & 1998 & 1999 & 2000 & Media \\
\hline 1 & 14.828 & 26.542 & 21.970 & 21.113 & 96,7 & 97,8 & 95,0 & 96,5 \\
\hline 2 & 19.513 & 27.770 & 24.056 & 23.780 & 98,2 & 97,9 & 94,0 & 96,7 \\
\hline 3 & 17.371 & 26.256 & 22.256 & 21.961 & 97,8 & 97,3 & 92,3 & 95,8 \\
\hline Media & $17.237 \mathrm{~b}$ & $26.856 a$ & $22.761 a$ & 22.285 & 97,7 & 97,7 & 93,8 & 96,3 \\
\hline
\end{tabular}

${ }^{1}$ Calibre $>70 \mathrm{~mm}$.

Dentro de cada fila y parámetro valores seguidos por diferentes letras indican diferencias entre años de acuerdo al test de Duncan $(P<0,05)$. 
Tabla 4. Peso medio del fruto y número de frutos por árbol para los diferentes tratamientos (número de goteros) durante el período experimental

Table 4. Average fruit weight and number of fruits per tree according to the different treatments (number of drippers) throughout the experimental period

\begin{tabular}{|c|c|c|c|c|c|c|c|c|}
\hline \multirow[b]{2}{*}{$\mathrm{N}^{\circ}$ goteros } & \multicolumn{4}{|c|}{ Peso (g) } & \multicolumn{4}{|c|}{ Cantidad (frut. árbol ${ }^{-1}$ ) } \\
\hline & 1998 & 1999 & 2000 & Media & 1998 & 1999 & 2000 & Media \\
\hline 1 & 136,4 & 183,6 & 195,2 a & 171,8 & 38 & 51 & 39 & 43 \\
\hline 2 & 139,0 & 212,6 & $191,7 \mathrm{ab}$ & 181,1 & 49 & 46 & 44 & 46 \\
\hline 3 & 139,7 & 206,1 & $189,1 \mathrm{~b}$ & 178,3 & 44 & 45 & 41 & 43 \\
\hline Media & $138,4 b$ & 200,8 a & 192,0 a & 177,1 & 44 & 47 & 41 & 44 \\
\hline
\end{tabular}

Dentro de cada columna, valores seguidos por diferentes letras indican diferencias de acuerdo al test de Duncan $(P<0,05)$. Dentro de cada fila y parámetro valores seguidos por diferentes letras indican diferencias entre años de acuerdo al test de Duncan $(P<0,05)$.

La escasa diferencia obtenida en las producciones entre los tratamientos puede atribuirse en parte a que un bajo porcentaje de raíces mojadas puede generar altos valores de evapotranspiración en manzano (Black y West, 1974) y afectar a su producción. Así pues, el tratamiento con 1 emisor/árbol y un $22 \%$ de volumen de raíces mojadas, pero con la mayor frecuencia de riego, pudo haber sido suficiente para alcanzar un alto coeficiente de transpiración y de producción que no fueron incrementados significativamente por los otros porcentajes. Además, como un bajo número de goteros por planta supone reducir la superficie mojada del suelo y como consecuencia reducir drásticamente la evaporación (Girona et al., 2008), en el tratamiento con 1 gotero por árbol podría haber permanecido más cantidad de agua a disposición de la planta y resultar más productiva esta situación. En el caso de los tratamientos con un mayor número de emisores por árbol se produciría un mayor solapamiento entre los bulbos de humedad, con lo que la extensión de la zona mojada sería mayor, si bien esta humedad estaría más alejada de la zona de mayor densidad radicular y por tanto no sería aprovechada con la misma eficiencia por la planta, además de producirse una mayor evaporación. La variación en las condiciones de humedad en el suelo que se generan al aplicar diferentes frecuencias de riego entre distintos porcentajes de volumen de suelo húmedo ha sido constatada por Levin et al. (1980) y su influencia en el nivel de evaporación superficial del área mojada por Meshkat et al. (2000).

Ucar et al. (2016) encontraron diferencias en la producción y calidad en manzanos cv 'Gala' en el primer y segundo año de cultivo al aplicar el agua de riego consumida desde capacidad de campo a los 3, 5, 7 y 10 días, pero a diferencia del presente trabajo el suelo era franco-arcilloso y la cantidad de agua aplicada fue distinta en cada tratamiento. La aplicación del mismo volumen de agua a todos los tratamientos durante la temporada de riego es un factor que necesariamente actúa homogeneizando los distintos tratamientos.

Finalmente, debe considerarse también que el portainjerto EM9 es enanizante, propio de las plantaciones intensivas, con un sistema radicular muy poco desarrollado y bastante superficial, que demanda una elevada frecuencia de riego (Rodríguez, 2004). 


\section{Crecimiento vegetativo}

Los árboles evaluados mostraron un desarrollo vegetativo similar durante el periodo experimental con una media de $8,5 \mathrm{~m}^{3}$ árbol $^{-1} \mathrm{y}$ desviación típica de $0,7 \mathrm{~m}^{3}$ árbol-1 $^{-1}$, lo cual indica que las diferencias en el volumen de copa fueron menores al $10 \%$. No hubo diferencias significativas al comparar el crecimiento medio anual de los brotes controlados entre los diferentes tratamientos en cada uno de los tres años, tampoco entre los valores medios finales del período analizado (Tabla 5). Cuando el contenido de agua en el suelo aumenta debido al riego y hay condiciones de alta carga de frutos, no hay respuesta de crecimiento vegetativo positivo por la mayor dominancia del fruto sobre los órganos vegetativos (Grossman y Dejong, 1994). Durante la cosecha de 1999, este comportamiento habitual no se observó, porque el mayor crecimiento vegetativo coincidió con la mayor producción por árbol. Esta respuesta, probablemente, sea debida a un proceso de alternancia productiva ya que 1998 fue un año con baja producción, que facilitó la acumulación de reservas para la cosecha del año siguiente.

\section{Parámetros de calidad del fruto}

No se encontraron diferencias significativas en la firmeza del fruto en relación con la cantidad de goteros instalados por árbol (Tabla 6). El resultado medio obtenido de firmeza del fruto, $62 \mathrm{Ncm}^{-2}$, es muy similar al obtenido por Rufat (2003), $63 \mathrm{Ncm}^{-2}$, comparando los sistemas de riego por goteo y micro-aspersión, que generaron diferentes volúmenes de suelo húmedo.

Los resultados relacionados con el contenido de almidón coinciden también, con las observaciones de Rufat (2003). El mayor contenido de almidón se obtuvo con el menor número de goteros y viceversa (Tabla 6). Las diferencias entre los tratamientos con 1 gotero por planta y 3 goteros por planta fueron significativas. Sin embargo no se encontró ninguna relación entre firmeza del fruto e índice de almidón para el conjunto de los tratamientos. Bouzo y Gariglio (2013) en un ensayo sobre índices de madurez con distintas variedades de manzana encontraron una relación negativa entre firmeza e índice de almidón aunque el ajuste mediante correlación lineal entre estas dos variables fue muy

Tabla 5. Crecimiento vegetativo anual del brote y productividad del agua de riego $\left(P_{\mathrm{a}}\right)$ para los diferentes tratamientos (número de goteros), durante el período experimental

Table 5. Vegetative shoot growth $(\mathrm{cm})$ and overall agronomic water-use efficiency $\left(P_{a}\right)$ for the different treatments (number of drippers) throughout the experimental period

\begin{tabular}{|c|c|c|c|c|c|c|c|c|}
\hline \multirow[b]{2}{*}{$\mathrm{N}^{\circ}$ goteros } & \multicolumn{4}{|c|}{ Crecimiento anual brote $(\mathrm{cm})$} & \multicolumn{4}{|c|}{$\mathrm{P}_{\mathrm{a}}\left(\mathrm{kg} \mathrm{m}^{-3}\right)$} \\
\hline & 1998 & 1999 & 2000 & Media & 1998 & 1999 & 2000 & Media \\
\hline 1 & 28,93 & 34,08 & 25,13 & 29,38 & 3,16 & 6,50 & 5,04 & 4,90 \\
\hline 2 & 28,12 & 32,33 & 24,07 & 28,17 & 4,35 & 6,83 & 5,56 & 5,80 \\
\hline 3 & 27,25 & 35,73 & 21,67 & 28,22 & 3,81 & 6,65 & 5,09 & 5,18 \\
\hline Media & $28,10 \mathrm{~b}$ & 34,05 a & $23,62 c$ & 28,59 & 3,77 & 6,66 & 5,23 & 5,22 \\
\hline
\end{tabular}

Dentro de cada columna, valores seguidos por diferentes letras indican diferencias de acuerdo al test de Duncan $(P<0,05)$. Dentro de cada fila y parámetro valores seguidos por diferentes letras indican diferencias entre años de acuerdo al test de Duncan $(P<0,05)$. 
Tabla 6. Firmeza del fruto e índice de almidón de acuerdo a los diferentes porcentajes de volumen de suelo húmedo durante el período experimental

Table 6. Fruit firmness and starch index according to the different percentages of wet soil volume throughout the experimental period

\begin{tabular}{llllllll}
\hline & \multicolumn{3}{c}{ Firmeza $\left(\mathrm{N} \mathrm{cm}^{-2}\right)$} & & \multicolumn{3}{c}{ Almidón ${ }^{1}$ (Nivel) } \\
\cline { 2 - 5 } \cline { 6 - 8 } N $^{\circ}$ goteros & 1999 & 2000 & Media & & 1999 & 2000 & Media \\
\hline 1 & 57,39 & 65,43 & 61,41 & & 9,1 & $6,2 \mathrm{a}$ & $7,7 \mathrm{a}$ \\
2 & 60,13 & 64,54 & 62,39 & & 8,9 & $5,7 \mathrm{ab}$ & $7,3 \mathrm{ab}$ \\
3 & 57,58 & 67,19 & 62,39 & & 8,9 & $4,9 \mathrm{~b}$ & $6,9 \mathrm{~b}$ \\
Media & $58,36 \mathrm{~b}$ & $65,72 \mathrm{a}$ & 62,06 & & $9,0 \mathrm{a}$ & $5,6 \mathrm{~b}$ & 7,3 \\
\hline
\end{tabular}

${ }^{1}$ Colorimetría de lodo.

Dentro de cada columna, valores seguidos por diferentes letras indican diferencias de acuerdo al test de Duncan $(P<0,05)$. Dentro de cada fila y parámetro valores seguidos por diferentes letras indican diferencias entre años de acuerdo al test de Duncan $(P<0,05)$.

bajo (coeficientes de determinación entre $0,04-0,20)$. Ajustes igualmente pobres también los encontraron Knee et al. (1989) y Blankenship et al. (1997) con manzana 'Fuji' $(0,26-0,34)$, si bien estos autores compararon la evolución de ambos parámetros con el desarrollo del fruto y en el presente ensayo se compararon los valores finales obtenidos en los distintos tratamientos. La diferencia entre el índice de almidón (IA) entre temporadas fue significativa y podría explicarse porque el IA es cualitativo, indicando presencia de almidón, pero no su concentración, por lo que para distintos IA, la cantidad de almidón presente en dos frutos puede ser la misma, además de que la hidrólisis del almidón está afectada por la temperatura (Moggia y Pereira, 2006). Finalmente la falta de tendencias claras en los valores entre firmeza e índice de almidón del ensayo también se deben al bajo número de valores comparados, que además, como casi todos los índices de maduración se caracterizan por una importante dispersión (Bouzo y Gariglio, 2013).

\section{Productividad del agua de riego}

La mayor productividad del agua de riego se produjo, cada año, en el riego con 2 goteros (media de 5,58 kg fruto $\mathrm{m}^{-3}$ agua de riego aplicada), pero no hubo diferencias significativas respecto a los otros tratamientos ( $\mathrm{Ta}$ bla 5). El valor medio de productividad del agua para el conjunto de los 3 años de ensayo (1998-2000) y los 3 tratamientos aplicados fue de 5,22 kg de fruta comercial por $\mathrm{m}^{3}$ de agua de riego aplicada, valor prácticamente equivalente al de $5,25 \mathrm{~kg} \mathrm{~m}^{-3}$ indicado por Clop et al. (2009) en manzano 'Golden Delicious' para el área de Lérida.

\section{Conclusiones}

El riego localizado en manzano cv 'Golden Delicious' cultivado en condiciones intensivas, con distinto número de emisores (1, 2 y 3 goteros por árbol) que se correspondieron a tres frecuencias de riego medias de 3, 6 y 9 días y 
a unos volúmenes de suelo húmedo aproximados de 22,44 y $66 \%$ del volumen potencial de exploración radicular, no mostró diferencias significativas en ningún parámetro productivo (producción, peso y número de frutos, crecimiento vegetativo) ni de calidad del fruto (firmeza, excepto contenido de almidón) analizados durante los tres años de duración del experimento. La productividad del agua tampoco presentó diferencias entre tratamientos. Probablemente la falta de diferencias significativas entre las variables analizadas pueda atribuirse principalmente a la aplicación del mismo volumen de agua entre tratamientos y al solapamiento entre bulbos de humedad que se produce en cultivos con reducido marco de plantación por la proximidad entre emisores y que define una franja contínua de humedad, factores que tienden a uniformizar los distintos tratamientos mediante un patrón de comportamiento hídrico parecido y de naturaleza compleja.

Por consiguiente, en plantaciones de alta densidad de manzanos (2.857 árboles ha-1), con condiciones de clima, suelo, riego y de calidad del agua similares a las del experimento, el establecimiento de 1 emisor por árbol tiene una ventaja competitiva importante respecto a otras configuraciones de riego basadas en un mayor número de goteros por árbol al producirse un costo económico de la instalación menor que en el diseño con 2 o 3 emisores, con producciones de rendimiento y calidad muy parecidas, al proporcionar la mayor frecuencia de riego en el caso de 1 gotero por planta un contenido de agua suficiente al volumen radicular desarrollado. Los resultados del trabajo sugieren recomendar la conveniencia de realizar un análisis detallado del proyecto mediante un número de goteros reducido con alta frecuencia de riego en la fase de diseño agronómico de las instalaciones de riego localizado en plantaciones de manzano intensivas, debido a las ventajas económicas que pueden derivarse de este diseño sin merma en la cantidad y calidad de la producción.
Finalmente, el ensayo permitió constatar la complejidad en la interacción entre las variables del riego tratadas (número de emisores, frecuencia y volumen de suelo mojado), los procesos de la dinámica del agua en el suelo y la respuesta productiva de las plantas cultivadas en condiciones de marcos de plantación intensivos, que probablemente obligan a definir criterios para el volúmen mojado óptimo distintos a los existentes para las plantaciones con marcos amplios.

\section{Agradecimientos}

Los autores agradecen al Ministerio de Economía y Competitividad la financiación de este trabajo mediante el proyecto HID19961342-C04-02 y al Instituto de Investigación y Tecnologías Agroalimentarias (IRTA), a través de la Estación Experimental Fundación Mas Badia (La Tallada d'Empordà, Gerona), el personal técnico y de soporte en campo aportado.

\section{Bibliografía}

Allen RG, Pereira LS, Raes D, Smith M (1998). Crop evapotranspiration. Guidelines for computing crop water requirements. FAO Irrigation and Drainage Paper No. 56. Roma, Italia. 299 p.

Amin MSM, Ekhmaj AIM (2006). DIPAC-drip irrigation water distribution pattern calculator. En: $7^{\text {th }}$ Micro-irrigation Congress, 16-15 de septiembre. PWTC, Kuala Lumpur, Malasia, p. 505-513.

Arbat G, Puig-Bargués J, Barragán J, Bonany J, Ramírez de Cartagena F (2008). Monitoring soil water status for microirrigation management versus modelling approach. Biosystems Engineering 100(2): 286-296.

Arbat G, Puig-Bargués J, Duran-Ros M, Barragán J, Ramírez de Cartagena F (2013). Drip-Irriwater: Computer software to simulate soil wetting patterns under surface drip irrigation. Computers and Electronics in Agriculture 98, 183-192. 
Black JDF, West DW (1974). Water uptake by an apple tree with various proportions of the root system supply with water. Proceedings of the Second International Drip Irrigation Congress, 7-14 de julio. San Diego, California, EE.UU. p. 432-433.

Blankenship SM, Parker M, Unrath CR (1997). Use of maturity indices for predicting poststorage firmness of 'Fuji' apples. Journal of Agricultural and Food Chemistry 45(1): 909-910.

Bouzo CA, Gariglio NF (2013). Validación de índices de cosecha para manzanas de bajos requerimientos de frío. Agrociencia Uruguay Vol. 17 (1): 74-80.

Clop M, Cots LI, Esteban M, Barragán JD (2009). Rentabilidad económica del regadío de los canales de Urgell (Lleida, España). ITEA-Información Técnica Económica Agraria 105(1): 36-48.

Doorenbos J, Pruitt WO (1988). Las necesidades de agua de los cultivos. FAO Serie Riego y Drenaje No. 24. Roma, Italia. 194 p.

Drake SR, Evans RG (1997). Irrigation management influence on fruit quality and storage life of 'Redspur' and 'Golden Delicious' apples. Fruit Varieties Journal 51(1): 7-12.

Earl KD, Jury WA (1977). Water movement in bare and cropped soil under insolate trickle emitters: II. Analysis of cropped soil experiments. Soil Science Society of America Journal 41: 856-861.

Fereres E (1981). Drip irrigation management. University of California. Leaflet No. 21259: 39 p.

Girona J, Mata M, Del Campo J, Marsal J (2008). Manejo del riego del manzano en condiciones de sequía extrema. Rural Cat, Dossier Tècnic $\mathrm{N}^{\circ}$ 30: 5-8.

Gispert JR, Ramírez de Cartagena F, Villar JM, Girona J (2012). Wet soil volume and strategy effects on drip-irrigated olive trees (cv. 'Arbequina'). Irrigation Science 31(3): 479-489.

Gispert JR, Ramírez de Cartagena F, Villar JM, Rovira M (2015). Efecto del volumen de suelo húmedo en riego localizado para el cultivo del avellano (Corylus avellana L.). ITEA-Información Técnica Económica Agraria 111(2): 109-126.
Grossman YL, Dejong TM (1994). Peach: A simulation model of reproductive and vegetative growth in peach trees. Tree Physiology 14: 329-345.

Kadayifçi A, Senyigit U, Dagdelen N, Öz H, Atilgan A (2010). The effects of different irrigation methods on root distribution, intensity and effective root depth of Young dwarf apple trees. African Journal of Biotechnology 9(27): 4217-4224.

Keller J, Bliesner RD (1990). Sprinkle and trickle irrigation. Van Nostrand Reinhold, Nueva York, EE.UU. 652 p.

Knee M, Hatfield SGS, Smith SM (1989). Evaluation of various indicators of maturity for harvest of apple fruit (Cox's Orange Pippin) intended for long-term storage. Journal of Horticultural Science 64(4): 403-411.

Levin I, Bravdo B., Assaf R (1973). Relation between apple root distribution and soil water extraction in different irrigation regimes. En: Physical aspects of soil water and salts in ecosystems (Ed. Hadas A et al.), p. 351-359, Vol 4, Series Ecological Studies. Springler-Verlag Berlín-Heildelberg, Alemania.

Levin I, Assaf R, Bravdo B (1979). Soil moisture and root distribution in an apple orchard irrigated by tricklers. Plant and Soil 52 (1): 31-40.

Levin I, Assaf R, Bravdo B (1980). Irrigation, water status and nutrient uptake in an apple orchard. En: Mineral Nutrition of Fruit Trees (Ed. Atkinson $D$ et al.), p. 255-264. Butterworths, Londrés, Reino Unido.

López JR, Hernández Abreu LM, Pérez Regalado A, González Hernández JF (1992). Riego localizado. Ed. Mundi Prensa, Madrid, España. 405 p.

Meshkat M, Wamer RC, Workman SR (2000). Evaporation reduction potential in an undisturbed soil irrigated with surface drip and sand tube irrigation. Transactions of ASAE 43(1): 79-86.

Moggia C, Pereira M (2006). Almidón y maduración en manzanas. Pomáceas. Boletín Técnico Vol. 6 (3). Universidad de Talca, Talca, Chile.

Neilsen GH, Parchomchuk P, Neilsen D, Berard R, Hague EJ (1995). Leaf nutrition and soil nutrients are affected by irrigation frequency and method for NP-fertigated 'Gala' apple. Journal of the American Society for Horticultural Science 120(6): 971-976. 
Oster JD, Wichelns D (2003). Economic and agronomic strategies to achieve sustainable irrigation. Irrigation Science 22(3): 107-120.

Raffo MD, Iglesias N (2004). Efecto de la intercepción y distribución de la radiación fotosintéticamente activa en manzanos cv Fuji bajo cuatro sistemas de conducción en alta densidad. RIA 33 (2): 29-42 INTA, Argentina.

Rodríguez RO (2004). Evaluación de portainjertos clonales de manzano. INTA Argentina, Presentaciones, 16 p. Disponible en:

http://inta.gob.ar/documentos/evaluacion-deportainjertos-clonales-en-manzanos (10 de noviembre de 2016).

Rufat J (2003). Influencia del riego y del abonado nitrogenado sobre el comportamiento vegetativo y productivo y su efecto en la calidad del fruto en manzano. Tesis Doctoral. Escola Tècnica Superior d'Enginyeria Agrària, Universitat de Lleida, Lérida, España.

Rumayor A, Bravo A (1991). Effects of three systems and levels of irrigating apple trees. Scientia Horticulturae 47: 67-75.

SAS Institute (1996). SAS User's Guide 1996 Edition. Raleigh.

Simunek J, Van Genuchten MT, Sejna M (2006). HYDRUS, software package for simulating the two and three dimensional movement of wa- ter, heat and solute transport in variably-saturated media. Technical Manual 1. PC Progress, Praga, República Checa.

Smith M (1988). Manual for CROPWAT version 5.2. FAO, Roma, Italia. 45 p.

Soil Survey Staff (2010). Keys to Soil Taxonomy, 11 th Ed. USDA-Natural Resources Conservation Service, Washington, DC., EE.UU.

Thalheimer M, Paoli N, Steinkeller M (2000). Experienze con ridotti regimi di irrigazione su melo 'Golden Delicious'. Rivista di frutticoltura e di ortofloricoltura 62(6): 76-82.

Ucar Y, Kadayifci A, Askin MA, Kankaya A, Senyigit $U$, Yildirim $F$ (2016). Effects of irrigation frequency on yield and quality parameters in apple c.v. 'Gala, Galaxy'. Erwerbs-Obstbau 58 (3): 169-175.

Zhao Z, Wang W, Wu Y, Huang X (2012). Yield and water use efficiency of pear trees under drip irrigation with different surface wetted percentages. International Journal of Agriculture \& Biology 14: 887-893.

Zur B (1996). Wetted soil volume as design objective in trickle irrigation. Irrigation Science 16: 101-105.

(Aceptado para publicación el 10 de noviembre de 2016) 